\title{
Article \\ A Method for Evaluating the Maximum Capacity of Grid-Connected Wind Farms Considering Multiple Stability Constraints
}

\author{
Xingyang $W u^{1, *}$, Feng Xue ${ }^{2}$, Yi Tang ${ }^{3}$ and Jianfeng Dai ${ }^{4}$ (D) \\ 1 College of Energy and Electrical Engineering, Hohai University, Nanjing 210098, China \\ 2 NARI Group Corporation (State Grid Electric Power Research Institute), Nanjing 211106, China; \\ xue-feng@sgepri.sgcc.com.cn \\ 3 School of Electrical Engineering, Southeast University, Nanjing 210096, China; Tangyi@seu.edu.cn \\ 4 College of AutomatioFn \& College of Artificial Intelligence, Nanjing University of Post and \\ Telecommunication, Nanjing 210023, China; daijianfeng@njupt.edu.cn \\ * Correspondence: wuxingyang@hhu.edu.cn; Tel.: +86-15-85-06-61-306
}

Citation: Wu, X.; Xue, F.; Tang, Y.; Dai, J. A Method for Evaluating the Maximum Capacity of Grid-

Connected Wind Farms Considering

Multiple Stability Constraints.

Electronics 2022, 11, 509. https://

doi.org/10.3390/electronics11040509

Academic Editor: Zbigniew

Leonowicz

Received: 7 January 2022

Accepted: 5 February 2022

Published: 9 February 2022

Publisher's Note: MDPI stays neutral with regard to jurisdictional claims in published maps and institutional affiliations.

Copyright: () 2022 by the authors. Licensee MDPI, Basel, Switzerland. This article is an open access article distributed under the terms and conditions of the Creative Commons Attribution (CC BY) license (https:/ / creativecommons.org/licenses/by/ $4.0 /)$.

\begin{abstract}
Boosting the capacity of grid-connected wind farms will greatly contribute to increasing the share of sustainable energy in the global generation mix. It is imperative to study the way to quantitatively assess the maximum capacity of grid-connected wind farms in combination with power system stability characteristics. In this work, a method to evaluate the maximum capacity of grid-connected wind farms considering the joint constraints of frequency and voltage stability is proposed based on the global intrinsic property of frequency stability and the local characteristic of voltage stability. Firstly, the maximum capacity of grid-connected wind farms in the power grid with high wind power penetration is assessed globally based on the frequency stability constraints, and then locally considering the voltage stability constraints of each local power grid. Further on, a quantitative method to evaluate the capacity of grid-connected wind farms is proposed based on the correlation between the local static voltage stability margin and the local capacity of grid-connected wind farms, as well as the global constraint of the maximum capacity of grid-connected wind farms. Finally, the effectiveness of the proposed method is verified by the simulation results of an actual regional power grid.
\end{abstract}

Keywords: maximum capacity of grid-connected wind farms; quantitative evaluation; frequency stability constraint; voltage stability constraint

\section{Introduction}

To mitigate global climate change and air pollution, sustainable energy generation has been expanded rapidly around the world. As one of the most promising sustainable energy options, wind power provides environmental benefits through negligible greenhouse gas emissions. The development of wind power has progressed steadily in recent years. By the end of 2020, the global installed capacity of wind power was approximately $743 \mathrm{GW}$, which makes it a considerable force in the electricity generation of modern power systems [1].

As the capacity of grid-connected wind farms increases, the frequency and voltage stability of the power grid shows a decreasing trend [2-5]. The current impact of wind farm integration on frequency stability is mainly studied in three aspects: wind power proportion, inertial response, and frequency regulation means. Wind power proportion is the main relevant factor for the level of the power system stability in the future, and the frequency stability problem constrains the capacity of grid-connected wind farms [6-10]. The diversity of the wind turbine types and the control methods complicates the inertial response of the power system after a disturbance. The inertial response characteristics of wind power vary under different control methods, including conventional control inertial response and 
virtual inertia [11-14]. The former provides weak inertia support to the power grid. In the latter, as current-source virtual inertia is actually power support, only voltage-source virtual inertia has the capability of increasing the system inertia level equivalently. The means of frequency regulation after wind farm integration are more diversified, including rational control of grid-connected inverters, load shedding, and joint operation with energy storage, etc. The impact of wind farm integration on voltage stability is studied in three main aspects: wind power proportion, disturbance size, and improvement measures. In the receiving-end systems with multi-infeed HVDC transmissions, the capacity of gridconnected wind farms is strongly limited by the voltage stability [15]. Both the static voltage stability under a small disturbance and the transient voltage stability under a large disturbance restrict the maximum capacity of grid-connected wind farms. Measures to improve the system voltage stability have been considered in the literature from a planning perspective, including the use of technical means, such as energy storage, synchronous compensator, and flexible DC.

From the analysis above, it can be seen that the frequency and voltage stability problems have become the main constraints limiting the maximum capacity of grid-connected wind farms. To study the maximum capacity of grid-connected wind farms that can be accommodated by a power grid with a high proportion of wind power, the problems of "determining the global capacity" and "evaluating the local capacity" need to be solved in turn. Solving the former problem means assessing the maximum capacity of grid-connected wind farms globally in the whole power grid. However, solving the latter requires not only assessing the maximum capacity of grid-connected wind farms of each local power grid but also quantitatively evaluating the capacity of grid-connected wind farms of each local power grid under the constraint of the maximum capacity of grid-connected wind farms of the whole power grid.

Currently, numerous scholars and engineers have studied how to assess the maximum capacity of grid-connected wind farms under stability constraints, including frequency stability constraints [5-10] and voltage stability constraints [15]. However, only a certain kind of stability constraint is considered in some research, which may lead to the following problems. On the one hand, if the maximum capacity of grid-connected wind farms of the whole power grid is assessed only considering the frequency stability constraints, the maximum grid-connected capacity limit of each local power grid may be ignored. When the capacity of grid-connected wind farms of a local power grid exceeds its limit, the local static voltage stability margin will be lower than the static voltage stability criterion. On the other hand, if the maximum capacity of grid-connected wind farms of each local power grid is assessed only considering the voltage stability constraints, the maximum grid-connected capacity limit of the whole power grid may be neglected. When the sum of the maximum capacity of grid-connected wind farms of each local power grid is greater than the maximum capacity of the whole power grid, the frequency stability will not meet the requirements of the frequency stability criteria. In other research, although both the frequency and voltage stability constraints are considered, the evaluation process only considers them in turn and does not attach importance to the correlation of the evaluation results under different constraints. For instance, if the sum of the maximum capacity of grid-connected wind farms of each local power grid is no more than the maximum capacity of the whole power grid, how to quantitatively assess the capacity of grid-connected wind farms of each local power grid is still an issue worthy of research.

To address these issues above, the overall scheme of evaluating the maximum capacity of grid-connected wind farms is introduced. Then, the specific contents and stability criteria of the frequency and voltage stability constraints considered in obtaining the maximum capacity of grid-connected wind farms of the whole power grid and each local power grid are elaborated. Afterward, under the constraint of the maximum capacity of grid-connected wind farms of the whole power grid, a quantitative evaluation method of the capacity of grid-connected wind farms of each local power grid is proposed based on the correlation between the local static voltage stability margin and the local capacity of grid-connected 
wind farms. Then, the entire evaluation process of the maximum capacity of grid-connected wind farms under the combined constraints of frequency and voltage stability is proposed. Finally, simulations are carried out to verify the effectiveness of the proposed method with an actual regional grid.

\section{Overall Scheme}

In this paper, the overall scheme of assessing the maximum capacity of grid-connected wind farms of a power grid is first introduced. The schematic diagram of the general idea is shown in Figure 1. A three-area power grid is taken as an instance. Suppose that local power grids A and B are integrated with wind farms. The evaluation process needs to address the following issues.

(a) Obtain the maximum capacity of grid-connected wind farms of the whole power grid. The solution proposed in this work is to assess the maximum capacity of gridconnected wind farms of the whole power grid $P_{w-w h o l e}$ based on the frequency stability constraints;

(b) Obtain the maximum capacity of grid-connected wind farms of each local power grid. The solution proposed is to evaluate the maximum capacity of grid-connected wind farms of the local power grids $\mathrm{A}$ and $\mathrm{B}$, expressed as $P_{w-A-\max }$ and $P_{w-B-\max }$, based on the voltage stability constraints. Thus, the sum of them is obtained as:

$$
P_{w-\text { local }-\max , \Sigma}=P_{w-A-\max }+P_{w-B-\max }
$$

(c) Quantitatively assess the capacity of grid-connected wind farms of each local power grid. The solution proposed is as follows: if the maximum capacity of grid-connected wind farms of the whole power grid is no less than the sum of the capacity of gridconnected wind farms of all local power grids, i.e., $P_{w-w h o l e} \geq P_{w-l o c a l-\max , \Sigma}$, then the local power grids can be integrated with wind farms according to their maximum capacity respectively. In Figure 1, it is supposed that $P_{w-A}$ and $P_{w-B}$ are the ultimately determined values of the capacity of grid-connected wind farms of the local power grids $\mathrm{A}$ and $\mathrm{B}$. Then the strategic decision can be made that $P_{w-A}=P_{w-A-\max }$ $P_{w-B}=P_{w-B-\max }$. In the opposite situation, i.e., $P_{w-w h o l e}<P_{w-l o c a l-\max , \Sigma}$, under the constraint of the maximum capacity of grid-connected wind farms of the whole power grid, the capacity of grid-connected wind farms of each local power grid needs to be quantitatively assessed based on the correlation between the local static voltage stability margin and the local capacity of grid-connected wind farms. That is, the sum of the capacity of grid-connected wind farms of all local power grids being equal to the maximum capacity of the whole power grid is seen as the constraint condition, and the sum of the static voltage stability margins of all local power grids reaching its maximum value is regarded as the optimization objective. At last, the quantitative assessment results of the capacity of each local power grid are solved. In Figure 1, for example, there are three different schemes in which the sum of the capacity of grid-connected wind farms of the local power grids $\mathrm{A}$ and $\mathrm{B}$ is equal to the maximum capacity of the whole power grid. It is assumed that $k_{A-S 1}$ and $k_{B-S 1}$ are the static voltage stability margins of the local power grids $A$ and $B$ in scheme 1 respectively. Similarly, $k_{A-S 2}$ and $k_{B-S 2}$ are the static voltage stability margins in scheme 2 , and $k_{A-S 3}$ and $k_{B-S 3}$ are the static voltage stability margins in scheme 3 . Then we compare the sums of the static voltage stability margins in all the schemes and choose the scheme with the largest sum as the ultimate strategic decision. 


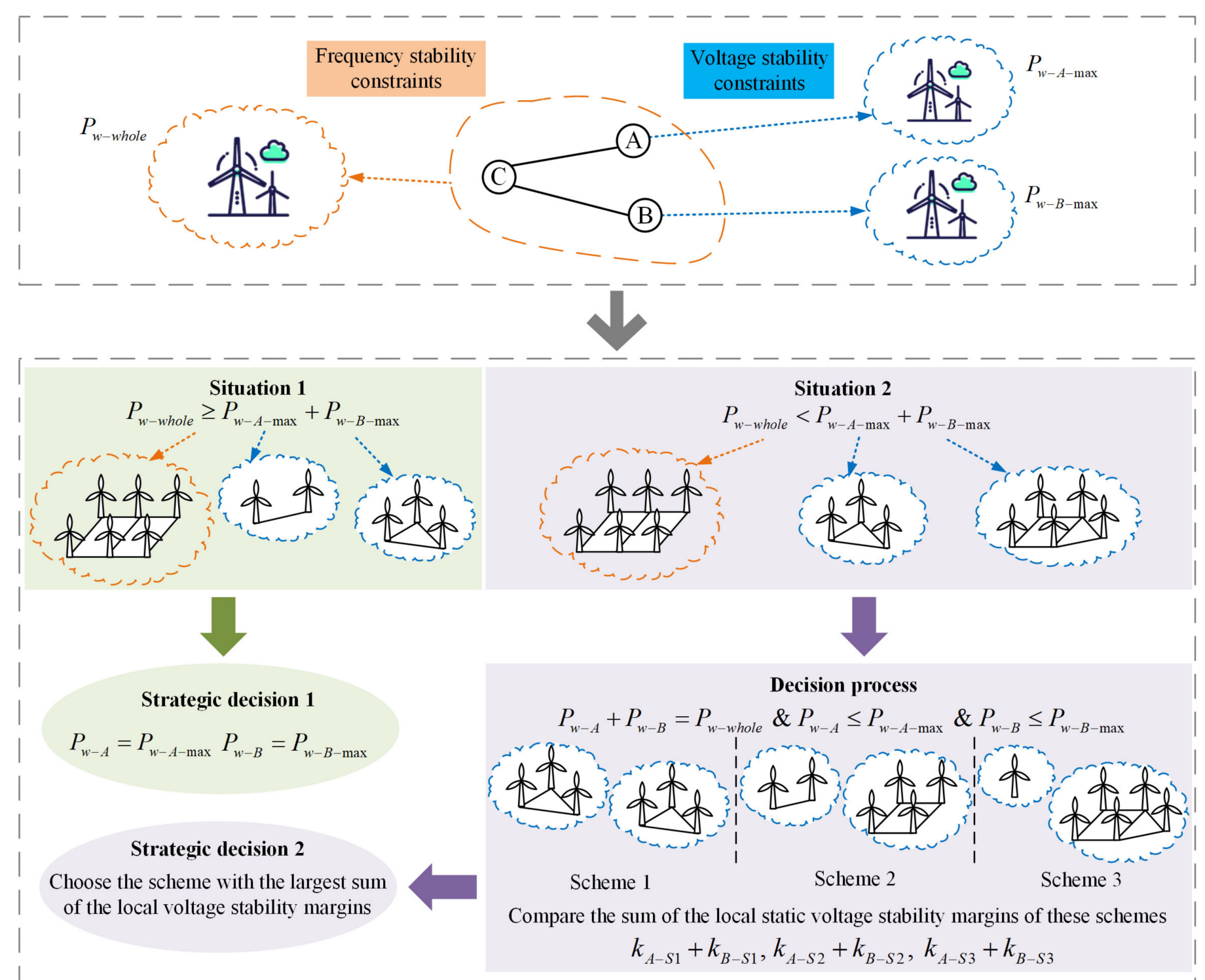

Figure 1. The overall scheme of assessing the maximum capacity of grid-connected wind farms of the power grid.

\section{Frequency and Voltage Stability Constraints}

\subsection{Frequency Stability Constraints}

Frequency stability refers to the ability of a power system to increase or decrease sufficient active power for regaining power balance after a high-power disturbance and to prevent frequency deviation from the nominal frequency of the system. The constraints associated with frequency stability include three aspects: rate of change of frequency (RoCoF), steady-state frequency deviation, and maximum transient frequency deviation.

\subsubsection{RoCoF Constraint}

RoCoF can be expressed as:

$$
\mathrm{RoCoF}=\frac{1}{2} \times \frac{\Delta P}{H} f_{n}
$$

where $\Delta P$ is the active power deficiency caused by the fault; $H$ is the inertia constant of the power system; $f_{n}$ is the nominal frequency of the power system. 
At the beginning of the power disturbance, the value of absolute RoCoF is the largest. If wind turbines are connected to the power grid replacing the original traditional synchronous generators, the effective inertia of the system will be reduced due to the decrease of the available effective kinetic energy of the power grid when the total installed capacity remains unchanged. In addition, even if the capacity of synchronous generators in the system remains unchanged, as the total installed capacity is increased by the wind farm integration, the effective inertia of the system will still decrease, for the reason that wind power generation provides almost no kinetic energy support to the power grid. When the active power deficiency remains unchanged, the decrease in the system inertia will lead to an increase in absolute RoCoF. Due to the tolerant ability of the equipment including underfrequency load shedding (UFLS) devices, synchronous generators, and new energy inverters to the maximum absolute RoCoF, the capacity of grid-connected wind farms is limited.

At present, there is no unified criterion for RoCoF constraint. RoCoF constraint is taken as a supplementary check constraint to other frequency stability constraints, and the check threshold value of the maximum absolute RoCoF is set at $1.176 \mathrm{~Hz} / \mathrm{s}$ [10] in the paper. That is, if the maximum value of absolute RoCoF does not exceed the check threshold value, the RoCoF constraint is no longer checked. Otherwise, it is necessary to check in detail according to the tolerant ability of various equipment of the studied power grid to the maximum absolute RoCoF.

\subsubsection{Steady-State Frequency Deviation Constraint}

When the system frequency tends to a steady state after the power system suffers an active power deficiency, the difference between the steady-state frequency and the nominal frequency is the steady-state frequency deviation.

Suppose the steady-state frequency deviation is $\Delta f$ after the active power deficiency $\Delta P$ occurs. The steady-state frequency deviation $\Delta f$ is compensated by the effect of load $\Delta P_{L}$ and the effect of synchronous generators $\Delta P_{G}$. The unit power regulation of load $K_{L}$ and the unit power regulation of synchronous generators $K_{G}$ can be represented as:

$$
\begin{gathered}
K_{L}=-\frac{\Delta P_{L} / P_{L 0}}{\Delta f / f_{N}}=-\frac{\Delta P_{L}}{P_{L 0}} \frac{1}{\Delta f_{*}} \\
K_{G}=-\frac{\Delta P_{G} / P_{G N}}{\Delta f / f_{N}}=-\frac{\Delta P_{G}}{P_{G N}} \frac{1}{\Delta f_{*}}
\end{gathered}
$$

where $P_{L 0}$ is the total active load at the nominal frequency; $f_{N}$ is the nominal frequency of the power system; $P_{G N}$ is the rated capacity of synchronous generators; $\Delta f_{*}$ is the per unit steady-state frequency deviation.

Then the unit power regulation of the power system $K_{S}$ on the basis of the total active load can be expressed as:

$$
K_{S}=-\frac{\Delta P}{P_{L 0}} \frac{1}{\Delta f_{*}}=-\frac{\Delta P_{L}+\Delta P_{G}}{P_{L 0}} \frac{1}{\Delta f_{*}}=\left(K_{L} \Delta f_{*}+K_{G} \Delta f_{*} \frac{P_{G N}}{P_{L 0}}\right) \frac{1}{\Delta f_{*}}=K_{L}+k_{r} K_{G}
$$

where $k_{r}=\frac{P_{G N}}{P_{L 0}}$ is the reserve coefficient, which represents the ratio of the rated capacity of synchronous generators to the total active load at the nominal frequency of the power system.

For the power system with integration of wind farms, if part of wind turbines can participate in primary frequency regulation, this influence needs to be considered in Equation (5) [16]. Then the unit power regulation of the power system $K_{S}$ needs to be revised as:

$$
K_{S}=K_{L}+k_{r}^{\prime} K_{G}+\rho K_{W F}
$$


where $k_{r}^{\prime}$ is the reserve coefficient of the power system with integration of wind farms; $\rho$ is the ratio of the rated capacity of wind turbines capable of primary frequency regulation to the total active load; $K_{W F}$ is the unit power regulation of wind turbines.

Thus the per unit steady-state frequency deviation can be expressed as:

$$
\Delta f_{*}=-\frac{\Delta P}{P_{L 0}} \frac{1}{K_{S}}=-\frac{\Delta P}{P_{L 0}} \frac{1}{K_{L}+k_{r}^{\prime} K_{G}+\rho K_{W F}}
$$

However, there are still many wind turbines without frequency regulation capability at present. For the sake of simplifying the analysis process, suppose that the wind turbines have no frequency regulation capability, i.e., $K_{W F}=0$. It can be inferred from Equation (7), if the load level is unchanged, the replacement of synchronous generators of the power grid by wind turbines decreases the value of $k_{r}^{\prime}$ and increases the absolute value of $\Delta f_{*}$. If the system's steady-state frequency deviation increases to a certain range, it has a negative impact on the power quality. Moreover, it increases the risk of frequency instability of the system after it suffers a large active power deficiency disturbance. According to the active power deficiency $\Delta P$ and the maximum acceptable steady-state frequency deviation, the startup quantity of synchronous generators $k_{r}^{\prime} P_{L 0}$ meeting the constraint of steady-state frequency deviation can be calculated. Further on, the maximum capacity of grid-connected wind farms is obtained as $\left(1-k_{r}^{\prime}\right) P_{L 0}$. The permissible range of the steady-state frequency deviation in the power grid of China is $\pm 0.2 \mathrm{~Hz}$, which is used as the stability criterion for the steady-state frequency deviation constraint in this paper.

\subsubsection{Maximum Transient Frequency Deviation Constraint}

When the system suffers the active power deficiency $\Delta P$, rotor motion state equation and simplified primary frequency regulation equation of thermal power units are as follows [16]:

$$
\left\{\begin{array}{l}
T_{J} \frac{d \Delta f_{*}}{d t}=\Delta M_{*} \approx \Delta P_{m}-\Delta P_{e}=\Delta P_{m}-\left(\Delta P-\Delta P_{L}\right) \\
\Delta P_{G}=\Delta P_{m}+\frac{d \Delta P_{m}}{d t} T_{C H}
\end{array}\right.
$$

where $T_{J}$ is rotor inertia time constant; $\Delta M_{*}$ is unbalanced torque; $P_{m}$ and $P_{e}$ are respectively mechanical power and electromagnetic power of rotor; $T_{C H}$ is high-pressure cylinder steam volume time constant.

Substitute Equations (3) and (4) into Equation (8), and conduct Laplace transformation, the following equations can be obtained:

$$
\left\{\begin{array}{l}
T_{J} \Delta f_{*}(s) s=\Delta P_{m}(s)-K_{L} \Delta f_{*}(s) P_{L 0}-\Delta P \\
-K_{G} \Delta f_{*}(s) P_{G N}=\Delta P_{m}(s)+\Delta P_{m}(s) T_{C H} s
\end{array}\right.
$$

Simultaneously solve equations in (9), the solution is:

$$
\Delta f_{*}(s)=-\frac{\Delta P\left(1+T_{C H} s\right)}{K_{G} P_{G N}+K_{L} P_{L 0}+K_{L} P_{L 0} T_{C H} s+T_{J} s+T_{J} T_{C H} s^{2}}
$$

Let $\eta=\sqrt{\left(T_{J}-K_{L} P_{L 0} T_{C H}\right)^{2}-4 K_{G} P_{G N} T_{J} T_{C H}}$ and conduct inverse Laplace transformation, $\Delta f_{*}(t)$ can be obtained as:

$$
\left\{\begin{array}{l}
\Delta f_{*}(t)=-C_{1} e^{-s_{1} t}-C_{2} e^{-s_{2} t} \\
s_{1}=\frac{-\left(T_{J}+K_{L} P_{L 0} T_{C H}\right)+\eta}{2 T_{J} T_{C H}} \\
s_{2}=\frac{-\left(T_{J}+K_{L} P_{L 0} T_{C H}\right)-\eta}{2 T_{J} T_{C H}} \\
C_{1}=\frac{\Delta P\left(T_{J}-K_{L} P_{L 0} T_{C H}+\eta\right)}{2 T_{J} \eta} \\
C_{2}=\frac{\Delta P\left(-T_{J}+K_{L} P_{L 0} T_{C H}+\eta\right)}{2 T_{J} \eta}
\end{array}\right.
$$


The maximum transient frequency deviation occurs when the power generation and the load of the system reach equilibrium for the first time after the fault of power deficiency. It is assumed that at the moment of $t_{e}, d f_{*} / d t=0$. Thus $t_{e}$ can be calculated as:

$$
t_{e}=\left(T_{J} T_{C H} / \eta\right) \ln \left[-C_{1} s_{1} /\left(C_{2} s_{2}\right)\right]
$$

Substitute Equation (12) into Equation (11), the maximum transient frequency deviation can be obtained as:

$$
\Delta f_{\max }\left(t_{e}\right)=-C_{1} e^{-s_{1} t_{e}}-C_{2} e^{-s_{2} t_{e}}
$$

With the active power deficiency and the system load level unchanged, the replacement of synchronous generators of the power grid by wind turbines makes the primary frequency regulation capability of the system weaker, which leads to a higher value of the maximum transient frequency deviation. The maximum transient frequency deviation determines whether the system will trigger the action of UFLS devices, which limits the maximum capacity of grid-connected wind farms. Thus the maximum transient frequency deviation criterion needs to be set in conjunction with the first-stage action frequency setting value of UFLS in the studied power grid.

\subsection{Voltage Stability Constraint}

Voltage stability includes static voltage stability, transient voltage stability, and mediumand long-term voltage stability. Since the studies in the field of medium- and long-term voltage stability are mainly about the voltage stability of the system under the action of dynamic elements and control devices with a slower response, medium- and long-term voltage stability is weakly associated with the wind farm integration. Only static voltage stability and transient voltage stability constraints are considered in this paper.

\subsubsection{Static Voltage Constraint}

(1) Influence of wind farm integration on static voltage stability of the PCC

After the integration of wind farms, the rest of the power system except wind farms can be equivalent to a series impedance of a voltage source when looking into the power system from a load node. Thus the equivalent model of the power grid integrated with wind power is shown in Figure 2 [17].

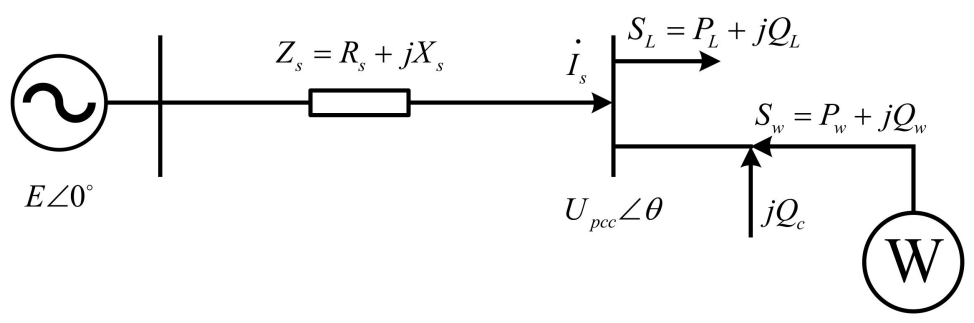

Figure 2. The equivalent model of the power grid integrated with wind power.

It is assumed that the Thevenin equivalent voltage of the rest of the power system is $E \angle 0^{\circ}$. The Thevenin equivalent impedance is $Z_{S}=R_{S}+j X_{S}$, where $R_{S}$ and $X_{S}$ are the Thevenin equivalent resistance and reactance respectively. The voltage of the point of common coupling (PCC) is $U_{p c c} \angle \theta$. The load apparent power is $S_{L}=P_{L}+j Q_{L}$, where $P_{L}$ and $Q_{L}$ are the active power and the reactive power of the load separately. The wind farm is regarded as a PQ node, of which the injected power is $S_{w}=P_{w}+j Q_{w} . P_{L}$ and $Q_{L}$ are the active power and the reactive power output of the wind farm separately. The reactive power $Q_{c}$ is sent out by the wind farm for centralized reactive power compensation.

The voltage of the PCC can be obtained as:

$$
\dot{U}_{p c c}=\dot{E}-\left(R_{s}+j X_{s}\right) \dot{I}_{s}
$$


Then the injected power into the power grid from the PCC can be expressed as:

$$
\left(P_{w}-P_{L}\right)+j\left(Q_{w}+Q_{c}-Q_{L}\right)=\dot{U}_{p c c} \cdot\left(\frac{\dot{U}_{p c c}-\dot{E}}{R_{s}+j X_{s}}\right)^{*}
$$

Further, the real part and the imaginary part can be represented as:

$$
\begin{gathered}
P_{w}-P_{L}=\frac{U_{p c c} E\left(X_{s} \sin \theta-R_{s} \cos \theta\right)+U_{p c c}^{2} R_{s}}{R_{s}^{2}+X_{s}^{2}} \\
Q_{w}+Q_{c}-Q_{L}=\frac{-U_{p c c} E\left(X_{s} \cos \theta+R_{s} \sin \theta\right)+U_{p c c}^{2} R_{s}}{R_{s}^{2}+X_{s}^{2}}
\end{gathered}
$$

By eliminating the phase angle difference $\theta$ between the Thevenin equivalent voltage and the voltage of the PCC, a unary quadratic equation about the PCC's voltage amplitude $U_{p c c}^{2}$ can be obtained as:

$$
\begin{aligned}
& U_{p c c}^{4}-\left[2 R_{s}\left(P_{w}-P_{L}\right)+2 X_{s}\left(Q_{w}+Q_{c}-Q_{L}\right)+E^{2}\right] U_{p c c}^{2} \\
& +\left(R_{s}^{2}+X_{s}^{2}\right)\left[\left(P_{w}-P_{L}\right)^{2}+\left(Q_{w}+Q_{c}-Q_{L}\right)^{2}\right]=0
\end{aligned}
$$

Then $U_{p c c}^{2}$ can be solved from Equation (18) as:

$$
U_{p c c}=\sqrt{B \pm \sqrt{B^{2}-C}}
$$

where:

$$
\begin{gathered}
B=R_{s}\left(P_{w}-P_{L}\right)+X_{s}\left(Q_{w}+Q_{c}-Q_{L}\right)+\frac{E^{2}}{2} \\
C=\left(R_{s}^{2}+X_{s}^{2}\right)\left[\left(P_{w}-P_{L}\right)^{2}+\left(Q_{w}+Q_{c}-Q_{L}\right)^{2}\right]
\end{gathered}
$$

It can be seen from Equations (19)-(21) that, $U_{p c c}$ is related to the network parameters and the active and reactive power injected by the PCC. Suppose the wind turbines operate in the unit power factor mode, thus $Q_{w}=0$. It is assumed that the values of $P_{L}, Q_{L}$, and $Q_{c}$ are small. When the wind power penetration is relatively high, i.e., $P_{w}$ is relatively large, $Q_{w}+Q_{c}-Q_{L}$ can be neglected compared to $P_{w}-P_{L}$. Thus the reactive power injected by the PCC is ignored [18]. Define the reference voltage and the reference capacity as $E$ and $E^{2} / X_{s}$. According to Equation (19), the relation between $P_{w}$ (p.u.) and $U_{p c c}$ (p.u.) is approximately obtained in the form of a PV curve which is similar to the PV curve of the load. With the increase in the active power output of the wind farm $P_{w}$, the PCC's voltage $U_{p c c}$ decreases gradually. At the time when $d P_{w} / d U_{p c c}=0$, a slight increase of $P_{w}$ will cause a significant change of the PCC's voltage, which might cause a voltage collapse.

\section{(2) The calculation method of the static voltage stability margin}

From the analysis above, it can be seen that the integration of wind farms is not conducive to the static voltage stability. After the wind farm integration in a certain area of the power grid, we make the load of the area grow with a constant power factor. When $d P / d V=0$, the active power of the load reaches its limit value $P_{L m a x}$. The point on the $P-V$ curve corresponds to the static voltage stability critical point. According to the Chinese power industry enterprise standard DL/T 1172-2013 “Guidelines for the Voltage Stability Evaluation of Power Systems", the static voltage stability margin $k$ can be expressed as:

$$
k=\frac{P_{L \max }-P_{L 0}}{P_{L 0}} \times 100 \%
$$

where $P_{L 0}$ is the initial active load. According to the previous analysis, as the capacity of grid-connected wind farms increases, the static voltage stability margin of the system decreases. 
The following criterion is given by DL/T 1172-2013 as well: under the maximum regional load or the maximum cross-sectional power flow, the active power margin of the regional load in normal operation or maintenance mode should be greater than $8 \%$. It is used as the stability criterion for the static voltage stability constraint.

\subsubsection{Transient Voltage Stability Constraint}

Wind turbines basically do not have voltage support capability. Although some wind farms are currently equipped with reactive power compensation of 10-60 Mvar, the reactive power support capability of the system is very weak when a severe fault occurs. In contrast, the maximum strong excitation of a thermal power unit can reach two times the rated voltage. It can be seen that after the replacement of synchronous generators in the power grid by wind turbines, the reactive support capacity during the faults will be greatly affected, which is not conducive to transient voltage stability.

According to the National Standard of the People's Republic of China GB 38755-2019 "Guidelines for Safety and Stability of Power Systems", the criterion for the transient voltage stability is that the load bus voltage can be restored to above the specified operating voltage level during the transient and dynamic processes after the power system is disturbed. The large disturbance is generally set to the $\mathrm{N}-1$ three-phase permanent fault set composed of all lines of the system. It should be noted that wind turbines have certain low-voltage ride-through capability and the fault duration is short. As long as the transient voltage is not destabilized, it will not cause the wind turbines to trip off.

However, the rapid development of the power grid makes the form of the power grid structure more complex. The probability of the simultaneous occurrence of the voltage instability and the power angle instability in the power system is high. The instability modes are coupled with each other. Although the theoretical and engineering criteria for the transient voltage instability and the power angle instability have been studied, these criteria can only identify a single instability phenomenon. The assessment of the maximum capacity of grid-connected wind farms considering the transient voltage stability constraint is faced with the problem that the coexistence with the power angle stability mode cannot be decoupled. The original intention of this paper is to propose a practical assessment method that is biased toward practical engineering applications, so the transient voltage stability constraint is not considered.

\subsection{Evaluation Results Based on the Constraints}

In order to obtain $P_{w-w h o l e}$, the quantity of wind turbines is incrementally added in the whole power grid by replacing synchronous generators until either steady-state frequency deviation $\Delta f_{\text {steady }}$ or the maximum transient frequency deviation $\Delta f_{\max }$ reaches its stability boundary. That is, $P_{w-w h o l e}$ is increased up to:

$$
\Delta f_{\text {steady }}=\Delta f_{\text {steady }-p} \text { or } \Delta f_{\max }=\Delta f_{\max -p}
$$

where $\Delta f_{\text {steady-p }}$ is the boundary of the stability criterion for the steady-state frequency deviation constraint; $\Delta f_{\max -p}$ is the boundary of the stability criterion for the maximum transient frequency deviation constraint.

It should be noted that the RoCoF constraint needs to be checked as a supplementary before $P_{w-w h o l e}$ is determined.

To assess the value of $P_{w-l o c a l-m a x, i}$ in turn, the quantity of wind turbines is incrementally increased in the $i$ th local power grid by replacing synchronous generators until the static stability margin $k_{l o c a l, i}$ reaches its stability boundary, which is expressed as:

$$
k_{l o c a l, i}=k_{p}
$$

where $k_{p}$ is the boundary of the stability criterion for the static voltage stability constraint. 


\section{Quantitative Assessment of the Capacity of Grid-Connected Wind Farms of Each Local Power Grid}

After obtaining $P_{w-w h o l e}$ under the frequency stability constraints and $P_{w-l o c a l-m a x, i}$, $P_{w-l o c a l-m a x, \Sigma}$ under the voltage stability constraints described in Section 3, the capacity of grid-connected wind farms of each local power grid needs to be quantitatively assessed. Still taking the three-area power grid in Figure 1 as an example, the quantitative assessment method is expounded as follows.

If $P_{w-w h o l e} \geq P_{w-l o c a l-m a x, \Sigma}$, then the local power grids $\mathrm{A}$ and $\mathrm{B}$ can be integrated with wind farms according to their respective maximum capacity of grid-connected wind farms $P_{w-A-\max }$ and $P_{w-B-\max }$.

Otherwise, the capacity of grid-connected wind farms of the local power grids A and B need to be quantitatively assessed. It is expected that the power grid stability characteristics will be maintained at a high level as far as possible in the evaluation scheme.

(1) The static voltage stability margins $k_{\text {local, } i}$ of each local power grid under different capacity values of $P_{w-l o c a l, i}$ are obtained by using the static voltage stability analysis module of the Power System Analysis Synthesis Program (PSASP) developed by the China Electric Power Research Institute. During the simulation, the adjusted power grid operating modes in accordance with the different capacity values of $P_{w-l o c a l, i}$ are the inputs for the static voltage stability analysis module of PSASP, while the corresponding static voltage stability margins $k_{\text {local }, i}$ are the outputs. After calculating multiple groups of data results of $k_{l o c a l, i}$ and $P_{w-l o c a l, i}$, the approximate relational expression between $k_{\text {local }, i}$ and $P_{w-l o c a l, i}$ can be obtained. Thus the approximate curves can be fitted. According to reference [19], the curves are similar to those shown in Figure 3.

(2) Set the constraint condition that the sum of the capacity of grid-connected wind farms of each local power grid is equal to the maximum capacity of the whole power grid, as expressed in Equation (25). Set the objective function that the sum of the static voltage stability margin of each local power grid reaches its maximum, as expressed in Equation (26).

$$
\begin{gathered}
P_{w-A}+P_{w-B}=P_{w-w h o l e} \\
F=\max \left(k_{A}+k_{B}\right)
\end{gathered}
$$

(3) Under the constraint condition of Equation (25), the objective function of Equation (26) is solved to obtain the quantitative assessment scheme of the capacity of grid-connected wind farms of the local power grids A and B respectively.

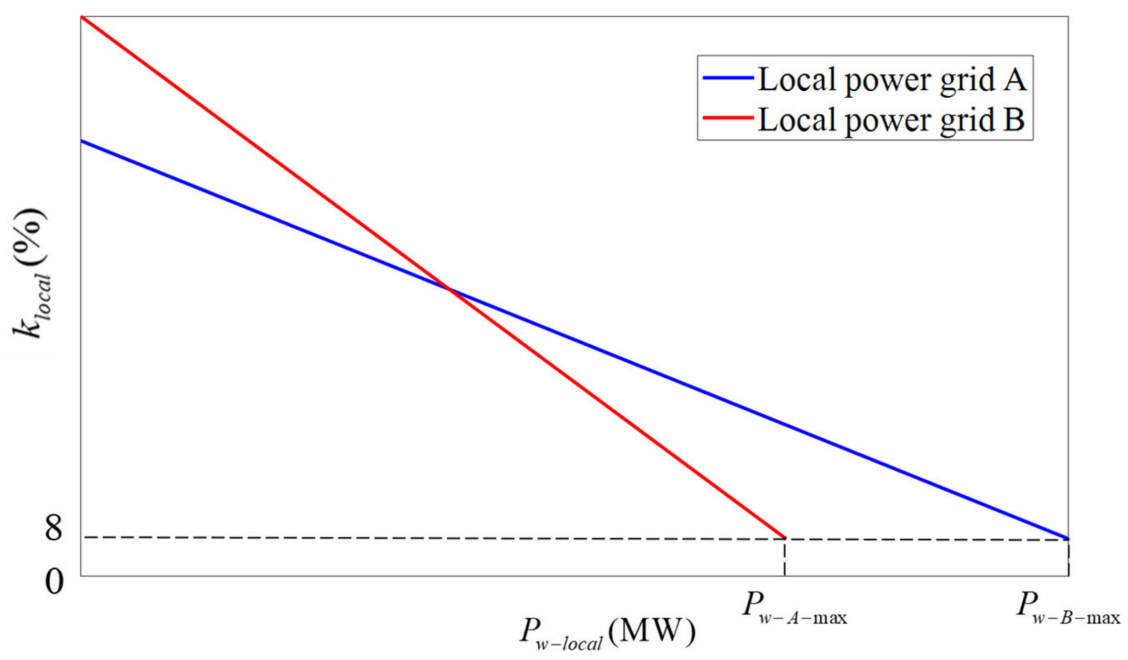

Figure 3. Approximate curves showing the relationship between $k_{\text {local }}$ and $P_{w-l o c a l}$. 


\section{Method for Assessing the Maximum Capacity of Grid-Connected Wind Farms under the Joint Constraints of Frequency and Voltage Stability}

Based on the analysis in Sections 2-4, the flowchart of the proposed method for assessing the maximum capacity of grid-connected wind farms under the joint constraints of frequency and voltage stability is shown in Figure 4.

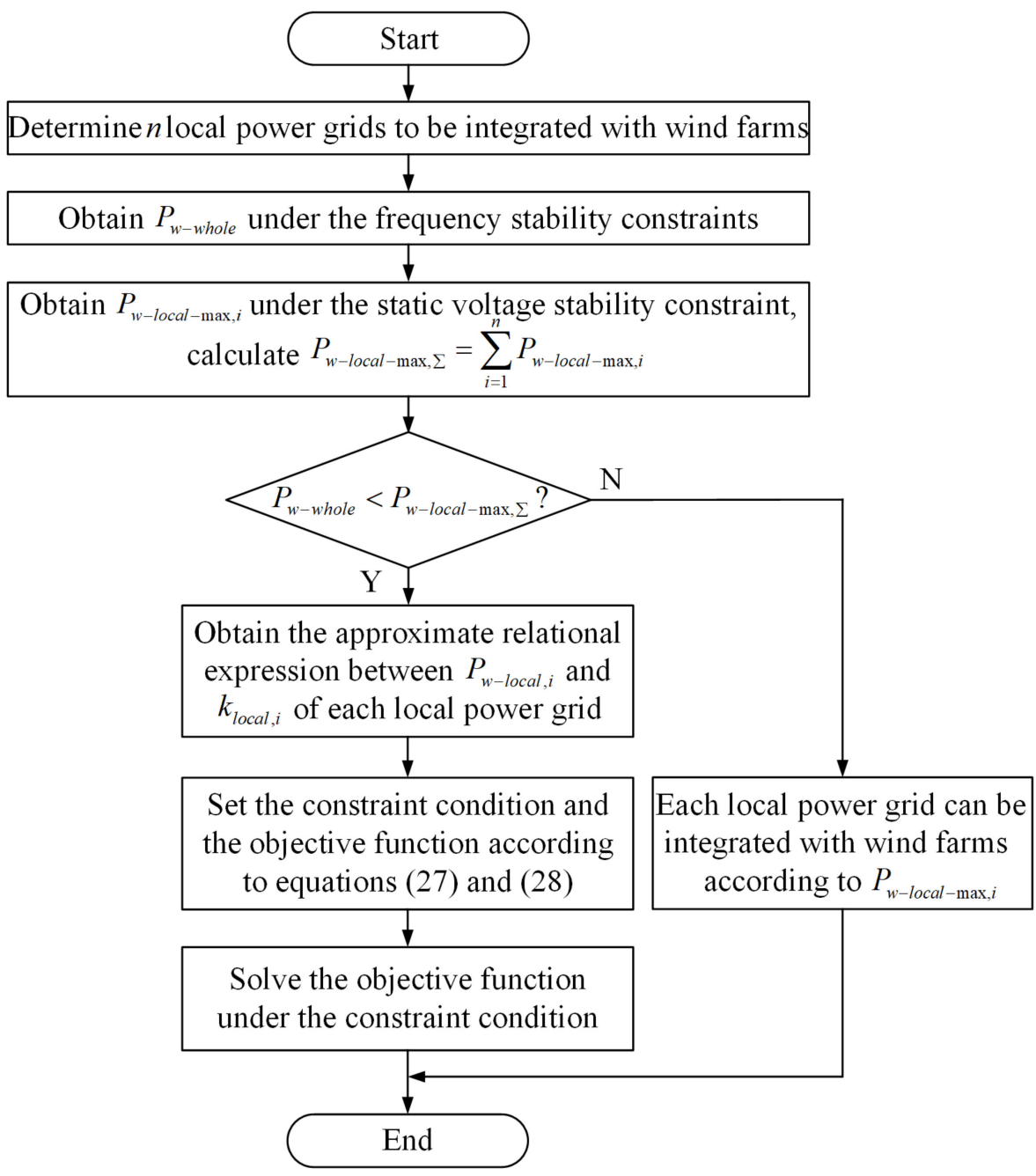

Figure 4. Flowchart of the proposed method for assessing the maximum capacity of grid-connected wind farms under the joint constraints of frequency and voltage stability.

Step 1: Determine $n$ local power grids to be integrated with wind farms. The way of wind farm integration is that wind turbines replace synchronous generators of equal capacity.

Step 2: Wind turbines are incrementally added to the whole power grid gradually, and set the tripping fault for the synchronous generator with the maximum output. Then use the transient stability program of PSASP for simulation calculations. When the system frequency just reaches the stability boundary of any one of the frequency stability constraints, take the capacity of grid-connected wind farms in this mode as the value of $P_{w-w h o l e}$.

Step 3: By using the static voltage stability analysis module of PSASP, the static voltage stability margin of each local power grid can be obtained separately. Gradually increase the quantity of wind turbines in the $i$ th local power grid until the static voltage stability margin reaches the stability boundary of $8 \%$, and a certain margin can be retained. The capacity of grid-connected wind farms in this mode is the value 
of $P_{w-l o c a l-m a x, i}$. Accomplish the simulations for all the local power grids, and the sum of $P_{w-\text { local-max }, i}$ can be obtained, i.e., $P_{w-l o c a l-\max , \Sigma}=\sum_{i=1}^{n} P_{w-\text { local-max }, i}$.

Step 4: If $P_{w-w h o l e} \geq P_{w-\text { local-max, } \Sigma}$, each local power grid can be integrated with wind farms according to $P_{w-l o c a l-\max , i}$.

Step 5: If $P_{w-w h o l e}<P_{w-l o c a l-m a x, \Sigma}$, the capacity of grid-connected wind farms of each local power grid needs to be quantitatively assessed. The static voltage stability margins $k_{\text {local }, i}$ of each local power grid under different capacity values of $P_{w-l o c a l, i}$ are obtained by using the static voltage stability analysis module of PSASP. Then the approximate relational expression between $k_{l o c a l, i}$ and $P_{w-l o c a l, i}$ can be obtained. Thus the approximate curves can be fitted.

Set the constraint condition and the objective function respectively as:

$$
\begin{gathered}
P_{w-\text { local }, \Sigma}=P_{w-\text { whole }} \\
F=\max \sum_{i=1}^{n} k_{\text {local }, i}
\end{gathered}
$$

Step 6: After solving the objective function under the constraint condition, quantitatively assessing the capacity of grid-connected wind farms of the whole and each local power grid is accomplished.

\section{Simulation Verification}

\subsection{Introduction to the Power Grid}

In this paper, simulation analysis is carried out based on the actual power grid data in Northwest China. The network topology of the power grid is shown in Figure 5. The total active load of the power grid is $1263 \mathrm{MW}$. The generator nodes are all conventional power sources with a total generation capacity of $1282 \mathrm{MW}$. Among them, the unit output of the $\mathrm{HB}$ thermal power plant is $150 \mathrm{MW}$, which is the largest in the whole power grid. It is assumed that the local power grids $A$ and $B$ are to be integrated with wind farms. Suppose that each wind turbine in the wind farms is a doubly-fed induction generator (DFIG)-based wind turbine with a capacity of 1.5 MW. The wind turbines do not participate in either frequency or voltage control. The local power grid A includes the generator nodes SM and KS as well as the load node KL with a load capacity of $180 \mathrm{MW}$. The local power grid B includes the generator nodes JE and JD as well as the load node LH with a load capacity of 122.5 MW. As the first-stage action frequency setting value of UFLS in the studied power grid is $49 \mathrm{~Hz}$, the stability criterion for the steady-state frequency deviation constraint is set as $0.8 \mathrm{~Hz}$, considering a margin of $0.2 \mathrm{~Hz}$.

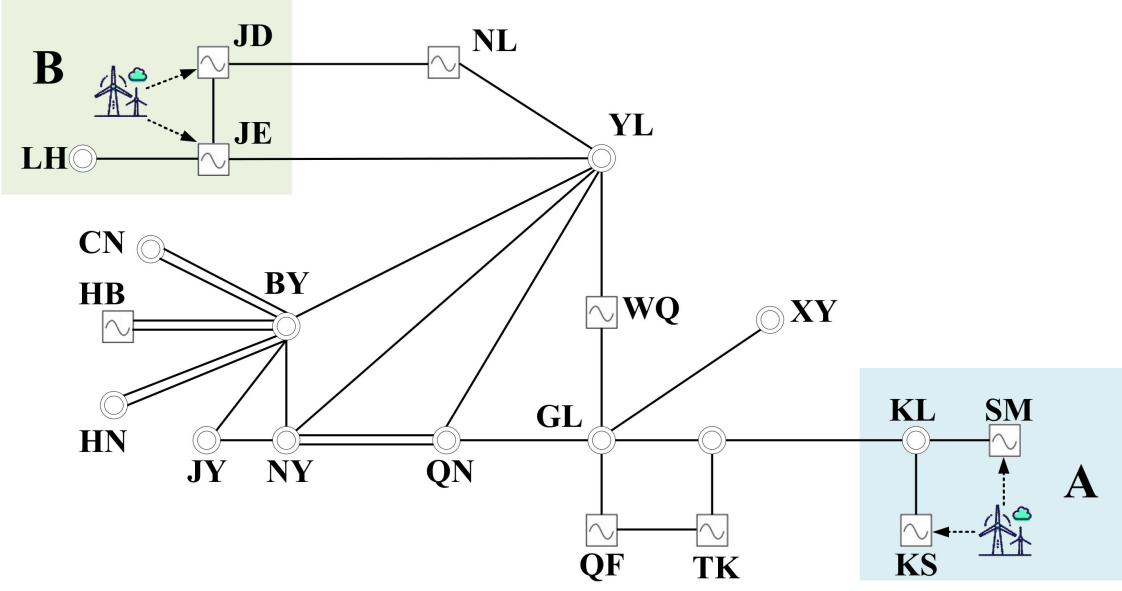

Figure 5. The network topology of the power grid. 


\subsection{Maximum Capacity of Grid-Connected Wind Farms of the Whole Power Grid}

The tripping of a $150 \mathrm{MW}$ thermal power unit in the HB plant is taken as the checking fault, which leads to a power deficiency ratio of $11.7 \%$. The capacity of wind power integration in the whole power grid varies from $0 \mathrm{MW}$ to $200 \mathrm{MW}$ with an incremental step size of $50 \mathrm{MW}$ approximately. The system frequency curves are shown in Figure 6a. Note from Figure 6a that the system frequency decreases with the increasing capacity of wind power integration. Specifically, the absolute values of the maximum transient frequency deviation, the steady-state frequency deviation, and RoCoF all show an increasing trend.

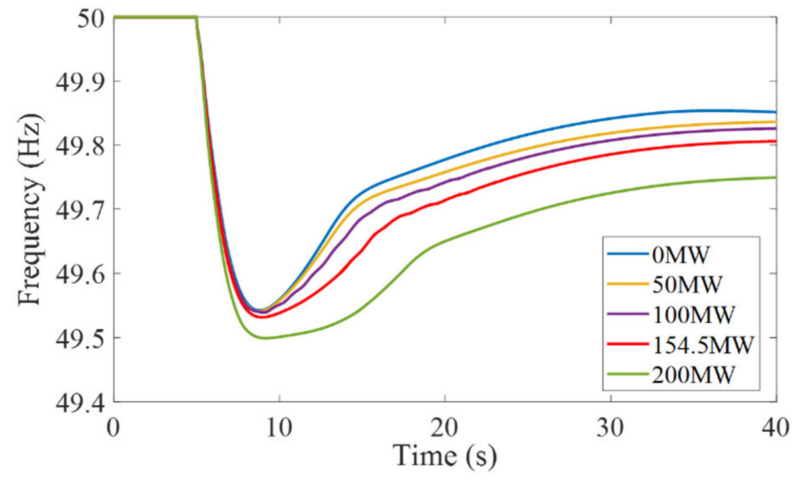

(a)

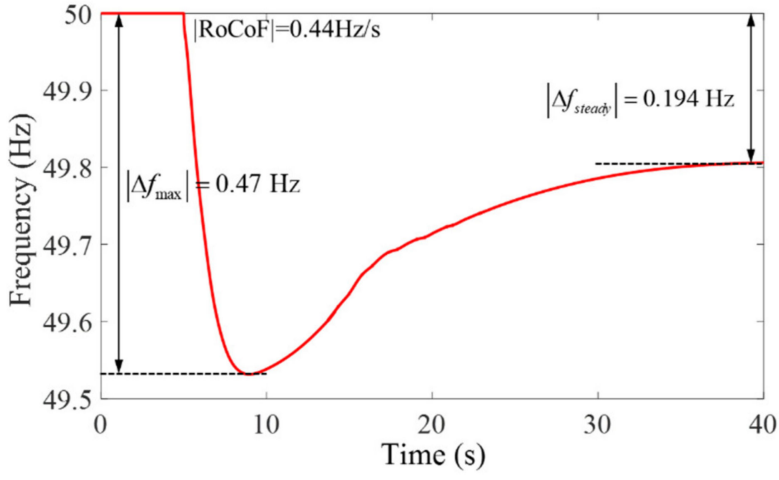

(b)

Figure 6. System frequency curves after one thermal unit in HB plant trips; (a) With increasing capacity of wind power integration; (b) With the wind power integration of 154.5 MW.

When the whole power grid is integrated with wind power of $154.5 \mathrm{MW}$ (the local power grid A with $50 \mathrm{MW}$, and the local power grid B with $104.5 \mathrm{MW}$ ) by replacing the synchronous generators in their respective areas, the system frequency curve is shown in Figure $6 \mathrm{~b}$ after the same fault occurs in this mode.

From Figure $6 \mathrm{~b}$, it is obvious that the system steady-state frequency deviation is $-0.194 \mathrm{~Hz}$, which is nearly at the boundary of the stability criterion for the steady-state frequency deviation constraint. Both $\mathrm{RoCoF}$ and the maximum transient frequency deviation meet the stability criteria. From the analysis above, it is clear that the maximum capacity of grid-connected wind farms of the whole power grid $P_{w-w h o l e}$ is $154.5 \mathrm{MW}$. The steady-state frequency deviation constraint is the main factor in determining $P_{w-w h o l e}$.

\subsection{Maximum Capacity of Grid-Connected Wind Farms of Each Local Power Grid}

By using the static voltage stability analysis module of PSASP for simulation, the PV curves and the static voltage stability margins of the local power grids A and B after the wind power integration are shown in Figure 7 and Table 1.

Table 1. Static voltage stability margins after the wind power integration.

\begin{tabular}{cccccc}
\hline Local Power Grid & $\begin{array}{c}\text { Capacity of } \\
\text { Grid-Connected } \\
\text { Wind Farms (MW) }\end{array}$ & Load Node & $\begin{array}{c}\text { Initial Load } \\
\boldsymbol{P}_{\mathbf{L} 0}(\mathbf{M W})\end{array}$ & $\begin{array}{c}\text { Limit Value of Load } \\
\boldsymbol{P}_{\text {Lmax }}(\mathbf{M W})\end{array}$ & $\begin{array}{c}\text { Static Voltage } \\
\text { Stability Margin } \boldsymbol{k}\end{array}$ \\
\hline \multirow{2}{*}{$\mathrm{A}$} & 70 & KL & 180 & 201.538 & $11.97 \%$ \\
& 76 & & 180 & 191.739 & $6.52 \%$ \\
B & 144.5 & LH & 122.52 & 134.035 & $9.40 \%$ \\
\hline
\end{tabular}




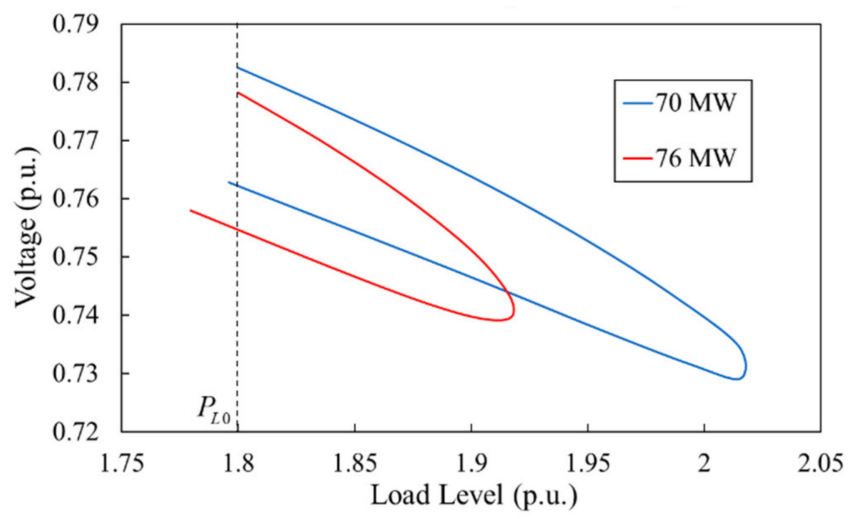

(a)

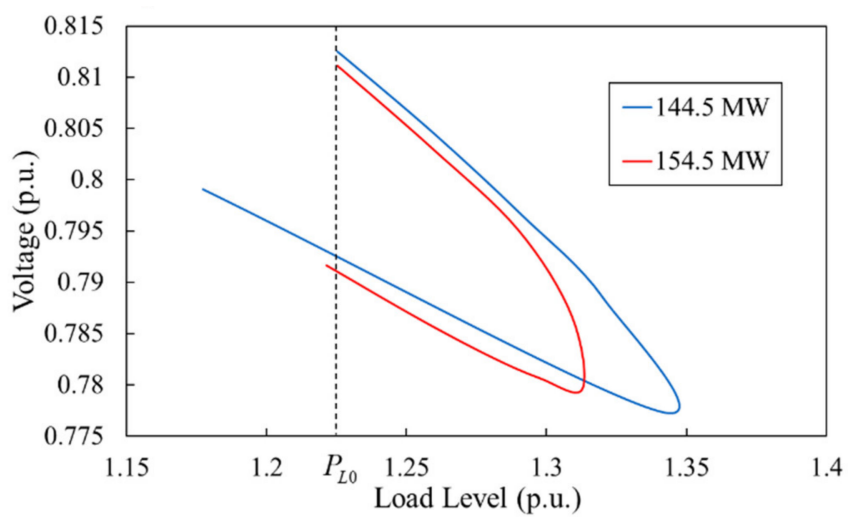

(b)

Figure 7. PV curves after the wind power integration in the local power grids A and B; (a) PV curves at the node $\mathrm{KL}$ in the local power grid A; (b) PV curves at the node LH in the local power grid $\mathrm{B}$.

From Table 1, it is obvious that the static voltage stability margin of the local power grid $\mathrm{A}$ is slightly higher than $8 \%$ after the wind power integration of $70 \mathrm{MW}$, while less than $8 \%$ after the wind power integration of $76 \mathrm{MW}$. With a certain margin considered, the maximum capacity of grid-connected wind farms of the local power grid A $P_{l o c a l-A-\max }$ is determined as $70 \mathrm{MW}$ in accordance with the static voltage stability criterion. Similarly, the maximum capacity of grid-connected wind farms of the local power grid $B P_{\text {local-B-max }}$ is 144.5 MW. The sum of $P_{\text {local }-A-\max }$ and $P_{\text {local }-B-\max }$ is $214.5 \mathrm{MW}$.

\subsection{Quantitative Assessment of the Capacity of Grid-Connected Wind Farms of Each Local Power Grid}

From the simulation analysis in Sections 6.2 and 6.3, it is evident that the maximum capacity of grid-connected wind farms of the whole power grid is less than the sum of the maximum capacity of grid-connected wind farms of the local power grids A and B, i.e., $P_{w-w h o l e}<P_{w-l o c a l-m a x, \Sigma}$. Consequently, the local power grids $\mathrm{A}$ and $\mathrm{B}$ cannot be integrated with wind power according to their respective maximum capacity. Further on, it is necessary to quantitatively assess the proper capacity of grid-connected wind farms of the local power grids $\mathrm{A}$ and $\mathrm{B}$.

The maximum capacity of grid-connected wind farms in the local power grid A is $70 \mathrm{MW}$ based on the static voltage stability criterion. Several values of the capacity are selected from 0 to $70 \mathrm{MW}$. The PV curves at the node KL in the local power grid A after the wind power integration of these capacity values above can be obtained by using the static voltage stability analysis module of PSASP. Moreover, the local static voltage stability margins corresponding to the PV curves are calculated. Then the approximate relational expression between $k_{A}$ and $P_{w-A}$ can be obtained and the approximate curve can be fitted. Similarly, simulations are carried out for the local power grid B. The PV curves and the fitted curves between $k_{\text {local }}$ and $P_{w-\text { local }}$ for the local power grids $\mathrm{A}$ and $\mathrm{B}$ are shown in Figures 8 and 9. As indicated in Figure $8 a, b$, the load limit decreases with the increasing capacity of wind power integration, leading to the decline of the static voltage stability margin.

The approximate relational expression between $k_{\text {local }}$ and $P_{w-l o c a l}$ can be expressed as:

$$
\left\{\begin{array}{c}
k_{A}=-0.4637 P_{w-A}+43.3964 P_{w-A} \in[0,70] \\
k_{B}=-0.2603 P_{w-B}+47.0019 P_{w-B} \in[0,144.5]
\end{array}\right.
$$

The constraint condition and the objective function can be illustrated as:

$$
\begin{gathered}
P_{w-A}+P_{w-B}=154.5 \\
F=\max \left(k_{A}+k_{B}\right)
\end{gathered}
$$


The relationship between $P_{w-A}$ and $k_{A}+k_{B}$ based on Equations (29)-(31) is shown in Figure 10.

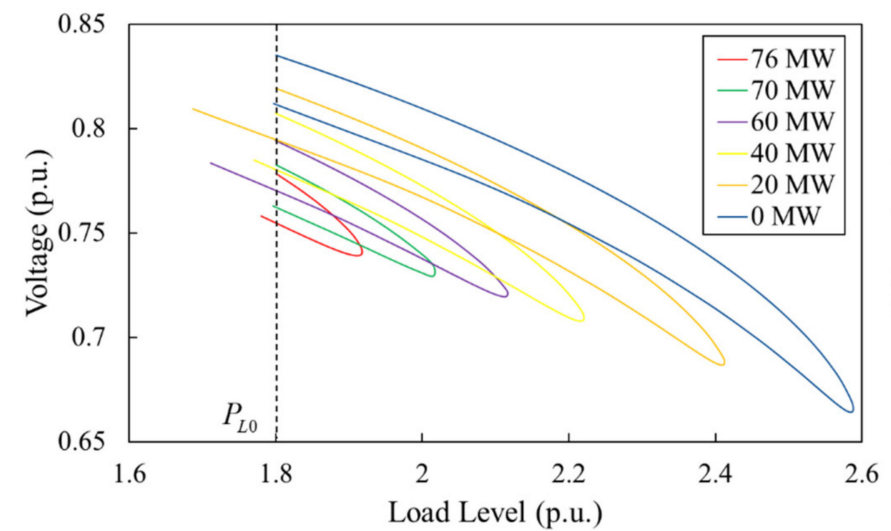

(a)

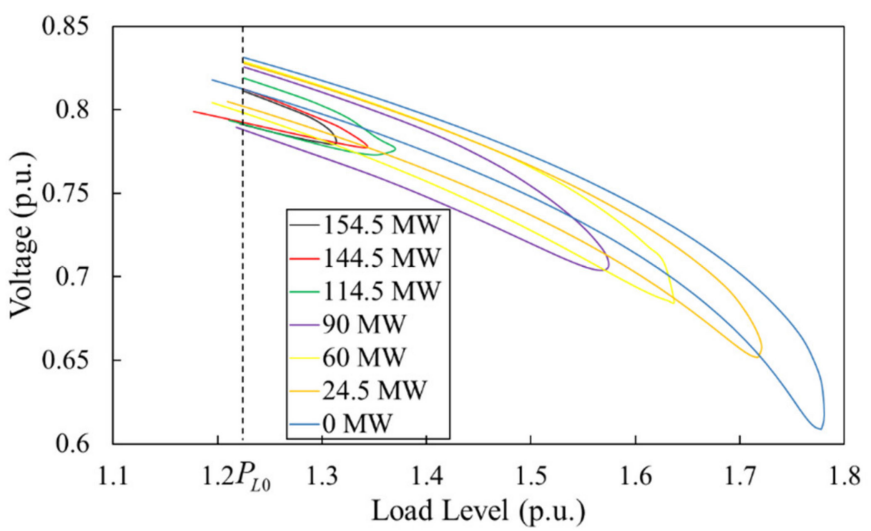

(b)

Figure 8. PV curves corresponding to different wind power integration capacity values in the local power grids A and B; (a) PV curves at the node KL in the local power grid A; (b) PV curves at the node LH in the local power grid B.

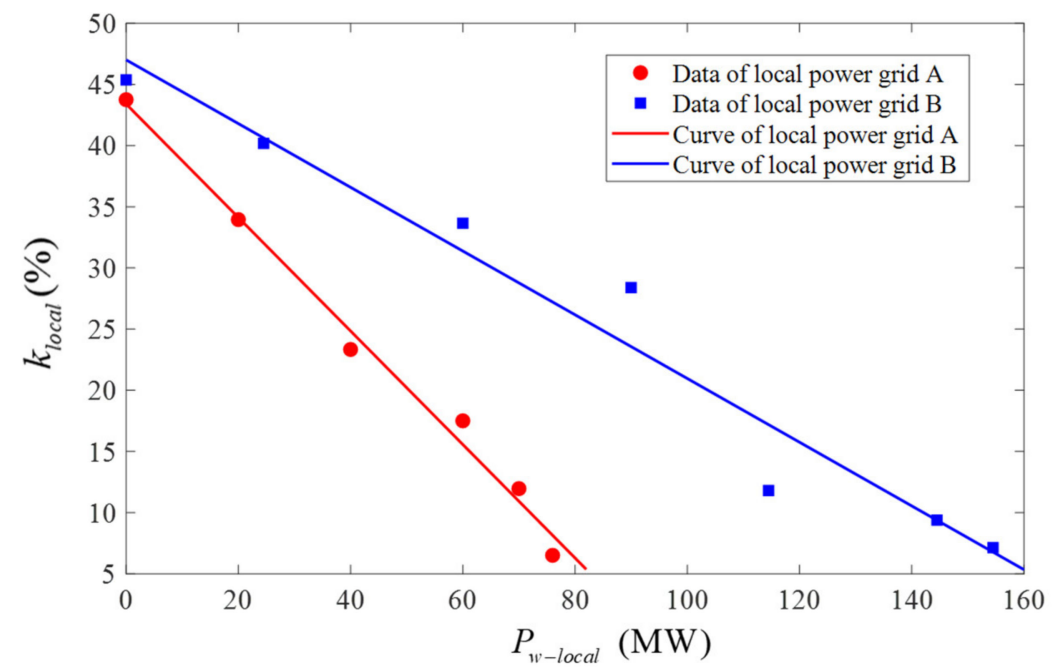

Figure 9. Approximate fitted curves of the relationship between $k_{\text {local }}$ and $P_{w-\text { local }}$.

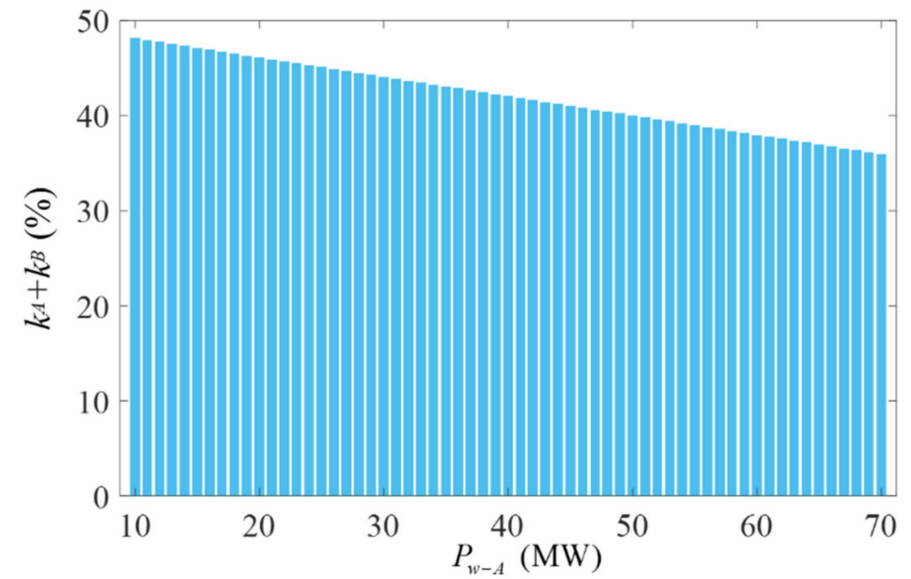

Figure 10. The relationship between $P_{w-A}$ and $k_{A}+k_{B}$. 
From Figure 10, it is obvious that $k_{A}+k_{B}$ shows a growing trend when $P_{w-A}$ declines but $P_{w-B}$ increases. In order to make $k_{A}+k_{B}$ reach the maximum value, the quantitative assessment result of $P_{w-A}$ and $P_{w-B}$ is as follows: the capacity of grid-connected wind farms of the local power grid $A$ is $10 \mathrm{MW}$ with its local static voltage stability margin of $38.76 \%$, while the capacity of the local power grid B is $144.5 \mathrm{MW}$ with its local static voltage stability margin of $9.40 \%$. Thus the sum of the static voltage stability margins of the local power grids $\mathrm{A}$ and $\mathrm{B}$ is taken as the maximum value of $48.16 \%$.

\subsection{Contrastive Analysis of the Proposed Method and Other Methods}

In reference [8], only the frequency stability constraints are considered to evaluate the maximum capacity of grid-connected wind farms of the whole power grid. Thus $P_{w-w h o l e}=154.5 \mathrm{MW}$ can be obtained by using the method proposed in reference [8]. Once the capacity of grid-connected wind farms of the local power grid A exceeds $70 \mathrm{MW}$, or the capacity of the local power grid B exceeds 144.5 MW, the static voltage stability criterion cannot be satisfied.

In reference [19], only the static voltage stability constraint is taken into account to assess the maximum capacity of grid-connected wind farms of the local power grids. Thus, $P_{w-A-\max }=70 \mathrm{MW}, P_{w-B-\max }=144.5 \mathrm{MW}$ can be obtained by using the method in reference [19]. Once the sum of the capacity of grid-connected wind farms of the local power grids A and B is more than $154.5 \mathrm{MW}$, the frequency stability criteria cannot be satisfied.

From the analysis above, it is clear that the method proposed in this article has the advantage of not only making both the frequency stability criteria and the static voltage stability criterion be satisfied but also maintaining the power grid stability characteristics at a high level as far as possible in the evaluation scheme.

It should be noted that this paper proposes a utility method for practical engineering applications. The function used to fit the curve is linear polynomial. Although there is a certain amount of error, the method has the advantage of being convenient in practical applications. What is more, the obtained quantitative assessment result not only meets the criteria for the frequency and voltage stability constraints of the power grid but also approaches the optimal solution in terms of the static voltage stability margin. Therefore, the method proposed in this paper is feasible and effective. It can provide a reference for the assessment of the capacity of grid-connected wind farms in actual projects.

\section{Conclusions}

In this paper, a method is proposed to assess the maximum capacity of grid-connected wind farms under the joint constraints of frequency and voltage stability. Taking the actual regional power grid as an example, the maximum capacity of grid-connected wind farms of the whole power grid under frequency stability constraints and of each local power grid under voltage stability constraints are obtained by simulation. Then the capacity of grid-connected wind farms of each local power grid is quantitatively assessed for the sake of maintaining a high level of the static voltage stability margin. The proposed method will provide technical support for the assessment of the capacity of grid-connected wind farms based on the power grid stability characteristics in actual engineering. The conclusions are as follows.

(1) A large-scale integration of wind farms will have adverse effects on both the frequency and voltage stability of the power grid. The frequency stability limits the maximum capacity of grid-connected wind farms of the whole power grid, and the voltage stability limits the maximum capacity of grid-connected wind farms of each local power grid. It is the theoretical basis of the method proposed in this paper.

(2) The quantitative assessment method proposed in this paper effectively solves the problem of how to quantitatively assess the capacity of grid-connected wind farms of each local power grid when the sum of the maximum capacity of grid-connected wind farms of each local power grid is greater than the maximum capacity of the 
whole power grid. Meanwhile, the static voltage stability margins of the local power grids can be maintained at a high level.

(3) In combination with the frequency and voltage stability problems mainly faced by modern power systems, the maximum capacity of grid-connected wind farms of the power grid is assessed. However, in some cases, the maximum capacity of gridconnected wind farms may also be constrained by other stability constraints, such as the thermal stability constraint, the power angle stability constraint, etc. What is more, there are other difficult issues in the research such as how to decouple the power angle instability mode and the transient voltage instability mode. In future work, the method for evaluating the maximum capacity of grid-connected wind farms including other stability constraints will be further studied.

\begin{abstract}
Author Contributions: Conceptualization, X.W. and Y.T.; methodology, F.X. and J.D.; software, X.W and J.D.; validation, X.W, and J.D.; formal analysis, F.X.; investigation, X.W. and J.D.; resources, Y.T.; data curation, X.W.; writing-original draft preparation, X.W.; writing-review and editing, Y.T. and J.D.; visualization, X.W.; supervision, F.X. All authors have read and agreed to the published version of the manuscript.
\end{abstract}

Funding: This research received no external funding.

Conflicts of Interest: The authors declare no conflict of interest.

\title{
References
}

1. Global Wind Report 2021. Available online: https://gwec.net/global-wind-report-2021/ (accessed on 25 March 2021).

2. Gevorgian, V.; Zhang, Y.; Ela, E. Investigating the impacts of wind generation participation in interconnection frequency response. IEEE Trans. Sustain. Energy 2017, 6, 1004-1012. [CrossRef]

3. Martínez-Lucas, G.; Sarasúa, J.I.; Pérez-Díaz, J.I.; Martínez, S.; Ochoa, D. Analysis of the implementation of the primary and/or inertial frequency control in variable speed wind turbines in an isolated power system with high renewable penetration. Case Study: El Hierro Power System. Electronics 2020, 9, 901. [CrossRef]

4. Dai, J.; Tang, Y.; Yi, J. Adaptive gains control scheme for PMSG-based wind power plant to provide voltage regulation service. Energies 2019, 12, 753. [CrossRef]

5. Nguyen, N.; Mitra, J. An analysis of the effects and dependency of wind power penetration on system frequency regulation. IEEE Trans. Sustain. Energy 2015, 7, 354-363. [CrossRef]

6. Yu, H.Y.; Bansal, R.C.; Dong, Z.Y. Fast computation of the maximum wind penetration based on frequency response in small isolated power systems. Appl. Energy 2014, 113, 648-659. [CrossRef]

7. Yan, R.; Saha, T.K.; Modi, N.; Masood, N.A.; Mosadeghy, M. The combined effects of high penetration of wind and pv on power system frequency response. Appl. Energy 2015, 145, 320-330. [CrossRef]

8. Dai, J.; Tang, Y.; Wang, Q. Fast method to estimate maximum penetration level of wind power considering frequency cumulative effect. IET Gener. Transm. Distrib. 2019, 13, 1726-1733. [CrossRef]

9. O'Sullivan, J.; Rogers, A.; Flynn, D.; Smith, P.; Mullane, A.; O'Malley, M. Studying the maximum instantaneous non-synchronous generation in an island system-frequency stability challenges in Ireland. IEEE Trans. Power Syst. 2018, 33, 985-994. [CrossRef]

10. Masood, N.A.; Yan, R.; Saha, T.K. A new tool to estimate maximum wind power penetration level: In perspective of frequency response adequacy. Appl. Energy 2015, 154, 209-220. [CrossRef]

11. Gil-González, W.; Montoya, O.D.; Escobar-Mejía, A.; Hernández, J.C. LQR-Based adaptive virtual inertia for grid integration of wind energy conversion system based on synchronverter model. Electronics 2021, 10, 1022. [CrossRef]

12. Li, T.; Wang, L.; Wang, Y.; Liu, G.; Zhu, Z.; Zhang, Y.; Zhao, L.; Ji, Z. Data-driven virtual inertia control method of doubly fed wind turbine. Energies 2021, 14, 5572. [CrossRef]

13. Xu, B.; Zhang, L.; Yao, Y.; Yu, X.; Yang, Y.; Li, D. Virtual inertia coordinated allocation method considering inertia demand and wind turbine inertia response capability. Energies 2021, 14, 5002. [CrossRef]

14. Krpan, M.; Kuzle, I. Dynamic characteristics of virtual inertial response provision by DFIG-based wind turbines. Electr. Power Syst. Res. 2020, 178, 106005. [CrossRef]

15. Vittal, E.; O'Malley, M.; Keane, A. A steady-state voltage stability analysis of power systems with high penetrations of wind. IEEE Trans. Power Syst. 2010, 25, 433-442. [CrossRef]

16. He, H.; Zhang, Y.; Sun, X.; Guo, Q.; Su, L.; Wang, Y.; Wang, M.; Huang, B. Evaluation method of renewable energy development scale and DC transmission scale of China Northwest power grid by considering frequency security constraints. Proc. CSEE 2021, $41,4753-4762$.

17. Burchett, S.; Douglas, D.; Ghiocel, S.G.; Liehr, M.; Chow, J.H.; Kosterev, D.; Faris, A.; Heredia, E.; Matthews, G.H. An optimal Thévenin equivalent estimation method and its application to the voltage stability analysis of a wind hub. IEEE Trans. Power Syst. 2018, 33, 3644-3652. [CrossRef] 
18. Tang, Y.; Chang, P.; Dai, J.; Yi, J.; Lin, W.; Yu, F. Evaluation method of maximum wind penetration level considering static voltage stability constraint. In Proceedings of the 2020 IEEE International Conference on Power Systems Technology (POWERCON), No.5, 11th Cross, 2nd Stage, West of Chord Road, Bangalore, Karnataka, India, 14-16 September 2020.

19. Yi, J.; Lin, W.; Yu, F.; Lin, A.; Yang, F. Calculation method of critical penetration of renewable energy constrained by static voltage stability. Power Syst. Tech. 2020, 44, 2906-2912. 